\title{
Reduced-Complexity Noncoherent Soft-Decision-Aided DAPSK Dispensing With Channel Estimation
}

\author{
Chao Xu, Student Member, IEEE, Dandan Liang, Student Member, IEEE, \\ Soon Xin Ng, Senior Member, IEEE, and Lajos Hanzo, Fellow, IEEE
}

\begin{abstract}
Differential amplitude phase-shift keying (DAPSK), which is also known as star-shaped quadrature-amplitude modulation, has implementational advantages not only due to dispensing with channel estimation but as a benefit of its low signal detection complexity as well. It is widely recognized that separately detecting the amplitude and the phase of a received DAPSK symbol exhibits lower complexity than jointly detecting the two terms. However, since the amplitude and the phase of a DAPSK symbol are affected by correlated magnitude fading and phase rotations, detecting the two terms completely independently results in a performance loss, which is particularly significant for soft-decision-aided DAPSK detectors relying on multiple receive antennas. Therefore, in this contribution, we propose a new soft-decision-aided DAPSK detection method, which achieves optimum DAPSK detection capability at substantially reduced detection complexity. More specifically, we link each a priori soft-input bit to a specific part of the channel's output, so that only a reduced subset of the DAPSK constellation points has to be evaluated by the soft DAPSK detector. Our simulation results demonstrate that the proposed soft DAPSK detector exhibits lower detection complexity than that of independently detecting the amplitude and the phase, whereas the optimal performance of DAPSK detection is retained.
\end{abstract}

Index Terms-Differential amplitude phase-shift keying (DAPSK), iterative demapping and decoding, Log-MAP, Max-Log-MAP, reduced complexity, soft-decision-aided detection, star quadrature-amplitude modulation (QAM).

\section{INTRODUCTION}

I T IS WIDELY recognized that soft-decision techniques are superior to hard-decision techniques. More explicitly, the classic quadrature-amplitude modulation (QAM) detector was further developed for processing soft bits, to show that the full potential of sophisticated coded modulation schemes can be beneficially exploited [1]. However, accurate channel state information (CSI) is required by coherent QAM detection for

Manuscript received May 13, 2012; revised January 31, 2013; accepted February 10, 2013. Date of publication February 14, 2013; date of current version July 10, 2013. This work was supported in part by the European Union's 7th Framework Programme (FP7/2007-2013) under the auspices of the CONCERTO project (288502), by the Engineering and Physical Sciences Research Council in the framework of the India-U.K. Advanced Technology Centre, and by the European Research Council Advanced Fellow Grant. The review of this paper was coordinated by Prof. H. H. Nguyen.

The authors are with the School of Electronics and Computer Science, University of Southampton, Southampton SO17 1BJ, U.K. (e-mail: cx1g08@ ecs.soton.ac.uk; d14e08@ecs.soton.ac.uk; sxn@ecs.soton.ac.uk; 1h@ecs. soton.ac.uk).

Digital Object Identifier 10.1109/TVT.2013.2247430 avoiding false phase locking of the carrier-recovery scheme [2]-[6]. As a remedy, differential amplitude phase-shift keying (DAPSK), which is also known as star QAM, was proposed in [7] to dispense with high-complexity CSI estimation and, hence, to eliminate the pilot overhead. It is demonstrated in [8] that low-complexity noncoherent schemes are particularly important in the context of relay-aided cooperative systems, where it is unrealistic to expect that the relay can altruistically estimate the source-relay channel.

The state-of-the-art channel decoding algorithms may be beneficially applied to the soft-decision-aided demodulators [1], [9]. More explicitly, the classic Bahl, Cocke, Jelinek, and Raviv algorithm in [10] invoked the MAP algorithm for channel decoding. Following the conception of the soft-output Viterbi algorithm [11] for reducing the complexity of the MAP algorithm, substantial research efforts have been dedicated to channel codes. An outstanding invention was the classic LogMAP algorithm [12], which operates the MAP algorithm in the logarithmic domain. The so-called Max-Log-MAP algorithm was also proposed in [12], which searched for the two maximum a posteriori symbol probabilities having their specific bit fixed to 1 and 0 , respectively. In this paper, we focus our attention on the low-complexity Max-Log-MAP conceived for soft-decision-aided DAPSK detection.

Apart from dispensing with channel estimation, DAPSK schemes also benefit from low signal detection complexity. It is widely recognized that separately detecting the amplitude and the phase of a received DAPSK symbol exhibits lower complexity than the complexity of jointly detecting the two terms. Following this idea, the hard-decision-aided and the softdecision-aided DAPSK demodulators were proposed in [7] and [13], respectively. However, in fact, the ring amplitude fading and the fading-induced phase rotations of a DAPSK symbol are correlated; hence, the attempt of detecting the two terms completely independently results in a performance loss, which is particularly significant for DAPSK detectors relying on multiple receive antennas. As a remedy, a novel soft-decision-aided DAPSK demodulator, which jointly detects the amplitude and the phase, was proposed in [14], but its detection complexity was substantially increased. Against this background, the novel contributions of this paper are as follows.

1) We demonstrate that a performance loss is imposed by independently detecting the received amplitude and the received phase of hard-decision-aided DAPSK, when 


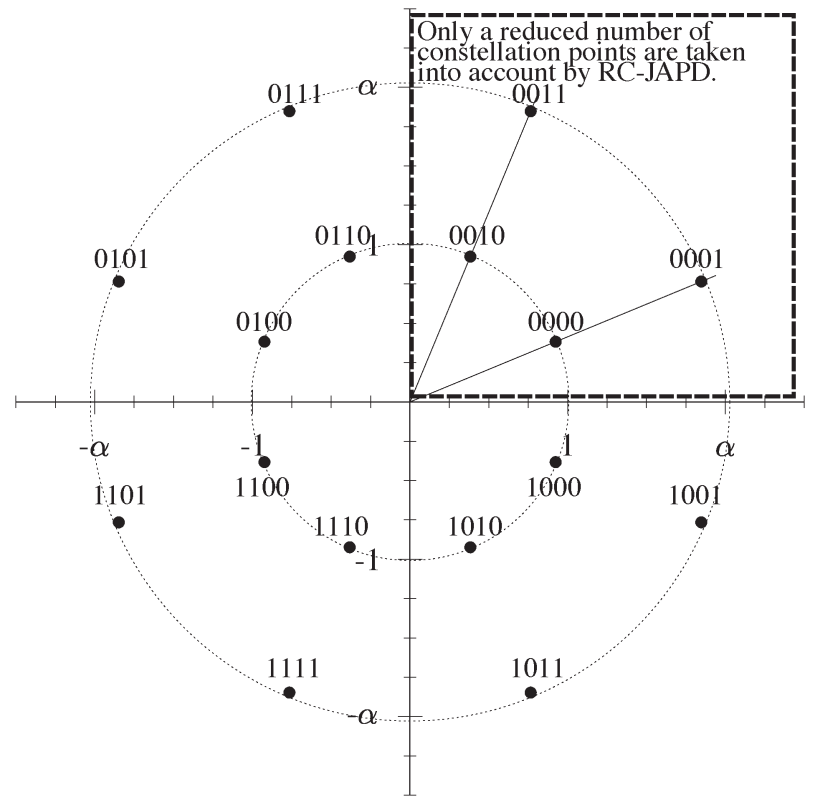

Fig. 1. Constellation diagram of 16-DAPSK $(2,8)$. The constellation diagram of $M$-DAPSK $\left(M_{A}, M_{P}\right)$ is rotated anticlockwise by $\pi / M_{P}$, so that there are exactly $M / 4$ constellation points in each quadrant.

multiple receive antennas are employed. As a remedy, we propose a new hard DAPSK detection method, which detects the amplitude with the aid of the detected phase. As a result of this "partially joint" amplitude-phase detection, optimal performance is retained.

2) Second, we propose a new method for substantially reducing the detection complexity of the soft-decisionaided DAPSK detector in [14], which may be invoked by a variety of iterative demapping and decoding schemes. More specifically, we link each a priori soft-input bit to a specific part of the channel's output, so that only a reduced subset of the DAPSK constellation points has to be evaluated by the soft DAPSK detector. Our simulation results demonstrate that the proposed soft DAPSK detector exhibits an even lower detection complexity than that of the separate amplitude-phase DAPSK detector in [13]. This is achieved without imposing any performance loss compared with the optimal joint amplitude-phase DAPSK detector in [14].

The remainder of this paper is organized as follows. The hard-decision-aided and the soft-decision-aided DAPSK detectors are introduced in Sections II and III, respectively. We provide simulation results in Section IV, and our conclusions are offered in Section V.

The following notations are used throughout this paper. $M$ DAPSK $\left(M_{A}, M_{P}\right)$ represents an $M$-DAPSK scheme that has $M_{A}$ ring amplitudes and $M_{P}$ phases, where we have $M=$ $M_{A} M_{P}$. The number of modulated bits per symbol is given by $\bar{m}=\bar{m}_{A}+\bar{m}_{P}$, where $\bar{m}_{A}$ and $\bar{m}_{P}$ are the bits that are mapped to the ring amplitude and to the phase, respectively. Furthermore, we use $(\cdot)^{*}$ to denote the conjugate of a complex symbol/vector, whereas $\|\cdot\|^{2}$ refers to the Euclidean norm of a vector/matrix. The subscript of a symbol (e.g., subscript $k$ in $s_{k}$ ) indicates the time index, whereas the superscript of a symbol (e.g., superscript $u$ in $s^{u}$ ) represents the modulation index.

\section{HARD-Decision-Aided DifFEREntial Amplitude Phase-Shift Keying Detection}

\section{A. DAPSK Modulation}

The constellation diagram of the classic 16-DAPSK $(2,8)$ scheme is portrayed in Fig. 1. We deliberately rotate all of the DAPSK constellations in [3], [7], [13], and [14] anticlockwise by $\pi / M_{P}$, so that there are exactly $M / 4$ constellation points in each quadrant. We will demonstrate in Section III that this feature is beneficial for our soft-decision-aided DAPSK detector design. Furthermore, Gray labeling is applied to all DAPSK schemes in this paper. The symmetry exhibited by the Gray-labeled DAPSK constellation diagram is the key to detection complexity reduction.

Similar to the regular QAM schemes [3], the power of the modulated DAPSK symbols has to be normalized. If we denote the ring ratio as $\alpha$, then the power normalization factor is given by $\left(\beta=\sum_{a=0}^{\left(M_{A}-1\right)} \alpha^{2 a} / M_{A}\right)$. Note that in Rayleigh fading channels, the advantageous choice for ring ratios are $(\alpha=2.0)$ for $M$-DAPSK $\left(2, M_{P}\right)[14]-[16]$ and $(\alpha=1.4)$ for $M$-DAPSK $\left(4, M_{P}\right)$ [17], [18], respectively.

If we denote the transmitted DAPSK symbol as $x_{k}=$ $\gamma_{k} \exp \left(j \psi_{k}\right)$, where $\gamma_{k}$ and $\psi_{k}$ refer to the amplitude and the phase, respectively, then differential encoding may be formulated as

$$
x_{k}= \begin{cases}\frac{1}{\sqrt{\beta}}, & \text { if } k=0 \\ s_{k-1} x_{k-1}, & \text { if } k>0\end{cases}
$$

where the modulated symbol $s_{k-1}=\gamma_{k} / \gamma_{k-1} \exp \left(j \Delta \psi_{k-1}\right)$ carries the source information. More explicitly, a generic DAPSK modulator assigns the first $\bar{m}_{P}$ bits to modulate the phase difference of the transmitted symbols $\omega_{k-1}=$ $\exp \left(j \Delta \psi_{k-1}\right)=\exp \left[j\left(\psi_{k}-\psi_{k-1}\right)\right]$ as an $M_{P}$-PSK phasor, whereas the last $\bar{m}_{A}$ bits are assigned to modulate the amplitude difference of the transmitted symbols $\rho_{k-1}=\gamma_{k} / \gamma_{k-1}$ based on the previous ring amplitude $\gamma_{k-1}$. More specifically, for tworing $M$-DAPSK $\left(2, M_{P}\right)$ schemes, the amplitude difference is modulated as

$$
\rho_{k-1}= \begin{cases}\alpha^{-1}, & \text { if } b_{\bar{m}}=1 \text { and } \gamma_{k-1}=\frac{\alpha}{\sqrt{\beta}} \\ 1, & \text { if } b_{\bar{m}}=0 \\ \alpha, & \text { if } b_{\bar{m}}=1 \text { and } \gamma_{k-1}=\frac{1}{\sqrt{\beta}}\end{cases}
$$

whereas for four-ring $M$-DAPSK $\left(4, M_{P}\right)$ schemes, the amplitude difference is modulated as

$$
\rho_{k-1}=\left\{\begin{array}{cl}
\alpha^{-3}, & \text { if } b_{\bar{m}-1} b_{\bar{m}}=01 \text { and } \gamma_{k-1}=\frac{\alpha^{3}}{\sqrt{\beta}} \\
\alpha^{-2}, & \text { if } b_{\bar{m}-1} b_{\bar{m}}=11 \text { and } \gamma_{k-1} \in\left\{\frac{\alpha^{2}}{\sqrt{\beta}}, \frac{\alpha^{3}}{\sqrt{\beta}}\right\} \\
\alpha^{-1}, & \text { if } b_{\bar{m}-1} b_{\bar{m}}=10 \text { and } \\
\alpha, & \text { if } b_{\bar{m}-1} \in\left\{\frac{\alpha}{\sqrt{\beta}}, \frac{\alpha^{2}}{\sqrt{\beta}}, \frac{\alpha^{3}}{\sqrt{\beta}}\right\} \\
& \text { if } b_{\bar{m}-1} b_{\bar{m}}=001 \text { and } \\
\quad \gamma_{k-1} \in\left\{\frac{1}{\sqrt{\beta}}, \frac{\alpha}{\sqrt{\beta}}, \frac{\alpha^{2}}{\sqrt{\beta}}\right\} \\
\alpha^{2}, \quad \text { if } b_{\bar{m}-1} b_{\bar{m}}=11 \text { and } \gamma_{k-1} \in\left\{\frac{1}{\sqrt{\beta}}, \frac{\alpha}{\sqrt{\beta}}\right\} \\
\alpha^{3}, \quad \text { if } b_{\bar{m}-1} b_{\bar{m}}=10 \text { and } \gamma_{k-1}=\frac{1}{\sqrt{\beta}} .
\end{array}\right.
$$


Since amplitude difference $\rho_{k-1}$ may be either equal to, smaller than, or larger than 1, depending on the previous ring amplitude $\gamma_{k-1}$, there are $\left(2 M_{A}-1\right)$ candidates for amplitude difference $\rho_{k-1}$ of an $M$-DAPSK $\left(M_{A}, M_{P}\right)$ scheme, as seen in (2) and (3).

\section{B. DAPSK Demodulation}

Let us now consider an uplink scenario, where the transmitter is equipped with a single antenna, whereas the receiver relies on $N_{R}$ receive antennas. For cooperative communication systems [8], [19], [20], $N_{R}$ may refer to the number of relay nodes. Due to the increasing number of virtual antenna array links and the requirement of imposing low signal processing complexity at the relay nodes, it becomes unrealistic to require accurate channel estimation; hence, DAPSK is preferred. The received signal of the $N_{R}$ receive antennas may be modeled as

$$
\mathbf{y}_{k}=x_{k} \mathbf{h}_{k}+\mathbf{n}_{k}
$$

where the $N_{R}$-element vectors $\mathbf{y}_{k}, \mathbf{h}_{k}$, and $\mathbf{n}_{k}$ model the received signal, the Rayleigh fading channels, and the additive white Gaussian noise, which has a zero mean and a variance of $N_{0}$ in each dimension, respectively. For quasi-static fading channels, we may assume $\left(\mathbf{h}_{k+1}=\mathbf{h}_{k}\right)$ over $T_{\mathrm{QS}}$ symbol periods. As a result, the next received signal vector may be expressed as

$$
\begin{aligned}
\mathbf{y}_{k+1} & =x_{k+1} \mathbf{h}_{k}+\mathbf{n}_{k+1} \\
& =s_{k} \mathbf{y}_{k}+\widetilde{\mathbf{n}}_{k+1}
\end{aligned}
$$

where the equivalent noise term $\left(\widetilde{\mathbf{n}}_{k+1}=-s_{k} \mathbf{n}_{k}+\mathbf{n}_{k+1}\right)$ is Gaussian distributed with a zero mean and a variance of $(1+$ $\left.\rho_{k}^{2}\right) N_{0}$ in each dimension. Therefore, the corresponding harddecision-aided DAPSK detection may be expressed as

$$
\hat{s}_{k}=\arg \min _{s^{u} \in \mathbf{s}}\left\|\mathbf{y}_{k+1}-s^{u} \mathbf{y}_{k}\right\|^{2}
$$

where $\left\{s^{u}\right\}_{u=1}^{\left(2 M_{A}-1\right) M_{P}}$ denotes the $u$ th element in the DAPSK symbol set $\mathbf{s}$. The demodulator of (6) operates on a vector-byvector basis, where the detection complexity is increased when the vector size is increased due to using more receive antennas. As a remedy, a decision variable may be introduced based on the Euclidean norm calculation of (6) as

$$
z_{k}=\mathbf{y}_{k+1} \cdot \mathbf{y}_{k}^{*} /\left\|\mathbf{y}_{k}\right\|^{2} .
$$

Naturally, minimizing metric $\left[\left\|\mathbf{y}_{k+1}-s^{u} \mathbf{y}_{k}\right\|^{2}=\left\|\mathbf{y}_{k+1}\right\|^{2}+\right.$ $\left.\left|s^{u}\right|^{2}\left\|\mathbf{y}_{k}\right\|^{2}-2 \operatorname{Re}\left\{\left(s^{u}\right)^{*} \mathbf{y}_{k+1} \cdot \mathbf{y}_{k}^{*}\right\}\right]$ in (6) and minimizing $\left[\left|z_{k}-s^{u}\right|^{2}=\left|z_{k}\right|^{2}+\left|s^{u}\right|^{2}-2 \operatorname{Re}\left\{\left(s^{u}\right)^{*} z_{k}\right\}\right]$ are equivalent because $\left\|\mathbf{y}_{k+1}\right\|^{2},\left\|\mathbf{y}_{k}\right\|^{2}$, and $\left|z_{k}\right|^{2}$ are all invariant over the different candidates $s^{u} \in \mathbf{s}$. Therefore, decision variable $z_{k}$ may be used for detecting the amplitude and the phase of $s_{k}=\rho_{k} \omega_{k}$ separately as

$$
\begin{aligned}
& \hat{\rho}_{k}=\min _{\rho^{v} \in \boldsymbol{\rho}}|| z_{k}\left|-\rho^{v}\right|^{2} \\
& \hat{\omega}_{k}=\min _{\omega^{l} \in \boldsymbol{\omega}}\left|z_{k}-\omega^{l}\right|^{2}
\end{aligned}
$$

where $\left\{\rho^{v}\right\}_{v=1}^{2 M_{A}-1}$ and $\left\{\omega^{l}\right\}_{l=1}^{M_{P}}$ denote the $v$ th element in ring amplitude subset $\rho$ and the $l$ th element in phasor subset

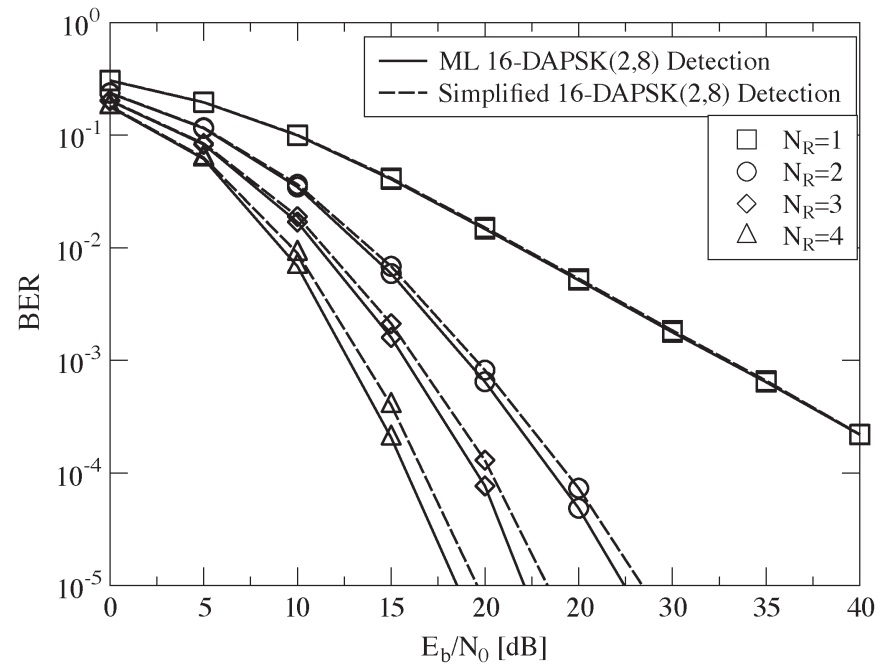

Fig. 2. Performance comparison between the ML DAPSK detecton of (6) and the simplified DAPSK detection of (8) and (9).

$\boldsymbol{\omega}$, respectively. For the special case of $\left(N_{R}=1\right)$, we have $\left|z_{k}\right|=\left|y_{k+1} \cdot y_{k}^{*}\right| /\left|y_{k}\right|^{2}=\left|y_{k+1}\right| /\left|y_{k}\right|$ and $\angle z_{k}=\angle z_{k} /\left|z_{k}\right|=$ $\angle\left(y_{k+1} \cdot y_{k}^{*}\right) /\left|y_{k+1} \cdot y_{k}^{*}\right|=\angle y_{k+1}-\angle y_{k}$. Therefore, (8) and (9) are equivalent to the hard-decision-aided star QAM detection introduced in [7].

However, the maximum-likelihood (ML) DAPSK detector of (6) and the simplified DAPSK detector of (8) and (9) do not have the same detection capability, as evidenced in Fig. 2, where the performance loss of (8) and (9) becomes significant as $N_{R}$ is increased. We will demonstrate in Section III that independently detecting the amplitude and the phase also results in substantial performance degradation for soft-decision-aided DAPSK detection relying on multiple receive antennas.

To elaborate a little further, the phase of a received DAPSK symbol may change the magnitudes on both the real and the imaginary axes of the received signal's constellation diagram, which implies that the detection of the amplitude, in fact, relies on the detection of the phase. Therefore, to restore the ML DAPSK detector's detection capability, we return to (6), which may be simplied as

$$
\begin{aligned}
\left\{\hat{\rho}_{k}, \hat{\omega}_{k}\right\} & =\arg \min _{s^{u} \in \mathbf{s}}\left|s^{u}\right|^{2}-2 \operatorname{Re}\left\{\left(s^{u}\right)^{*} z_{k}\right\} \\
& =\arg \min _{\rho^{v} \in \boldsymbol{\rho}, \omega^{l} \in \boldsymbol{\omega}}\left(\rho^{v}\right)^{2}-2 \rho^{v} \operatorname{Re}\left\{\left(\omega^{l}\right)^{*} z_{k}\right\} .
\end{aligned}
$$

We define the local minimum metric of $\left[\left(\rho^{v}\right)^{2}-\right.$ $\left.2 \rho^{v} \operatorname{Re}\left\{\left(\omega^{l}\right)^{*} z_{k}\right\}\right]$ in (10) as the minimum over the set of phasors $\omega^{l} \in \omega$ only, then (10) may be transformed to

$$
\begin{aligned}
\hat{\omega}_{k} & =\arg \min _{\omega^{l} \in \boldsymbol{\omega}}\left(\rho^{v}\right)^{2}-2 \rho^{v} \operatorname{Re}\left\{\left(\omega^{l}\right)^{*} z_{k}\right\} \\
& =\arg \min _{\omega^{l} \in \boldsymbol{\omega}}-\operatorname{Re}\left\{\left(\omega^{l}\right)^{*} z_{k}\right\}
\end{aligned}
$$

where a fixed amplitude is chosen from $\rho^{v} \in \boldsymbol{\rho}$. After deleting the constants in (9), it can be seen that (11) and (9) have become equivalent. The global minimum in (10) may now be obtained by comparing the local minimum metrics, which may be expressed as

$$
\hat{\rho}_{k}=\arg \min _{\rho^{v} \in \boldsymbol{\rho}}\left(\rho^{v}\right)^{2}-2 \rho^{v} \operatorname{Re}\left\{\left(\hat{\omega}_{k}\right)^{*} z_{k}\right\}
$$


where $\hat{\omega}_{k}$ is obtained by the local minimum search. As a result, (9) and (12) have exactly the same detection capability as (6). We have arranged for both detectors to process the same channel output, and they always produce the same decision. Moreover, (9) and (12) separately evaluate the phasor subset and the amplitude subset; hence, the low DAPSK detection complexity is retained.

\section{Soft-Decision-Aided Differential Amplitude PhASE-SHIFT KeYING DETECTION}

\section{A. Conventional Soft-Decision-Aided DAPSK Detection}

Here, we briefly summarize the features of the conventional soft-decision-aided DAPSK detectors, namely, those of the joint amplitude-phase detector (JAPD) in [14] and those of the separate amplitude-phase detector (SAPD) ${ }^{1}$ in [13].

The Log-MAP algorithm invoked by the JAPD produces the a posteriori log-likelihood ratio (LLR) $L_{p}\left(b_{m}\right)$ as [12], [21]

$$
L_{p}\left(b_{m}\right)=\ln \frac{\sum_{s_{k} \in \mathbf{s}_{b_{m}=1}} \exp \left[d\left(\rho^{v}, \omega^{l}\right)\right]}{\sum_{s_{k} \in \mathbf{s}_{b_{m}=0}} \exp \left[d\left(\rho^{v}, \omega^{l}\right)\right]}
$$

where $\mathbf{s}_{b_{m}=1}$ and $\mathbf{s}_{b_{m}=0}$ represent symbol set $\mathbf{s}$, when the specific bit $b_{m}$ is fixed to 1 and 0 , respectively, whereas JAPD's probability metric $d\left(\rho^{v}, \omega^{l}\right)$ is defined as [14]

$$
d\left(\rho^{v}, \omega^{l}\right)=-\frac{\left\|\mathbf{y}_{k+1}-\rho^{v} w^{l} \mathbf{y}_{k}\right\|^{2}}{\widetilde{N}_{0}^{v}}+\sum_{\widetilde{m}=1}^{\bar{m}} b_{\widetilde{m}} L_{a}\left(b_{\widetilde{m}}\right)
$$

where we have the equivalent noise power of $\left\{\tilde{N}_{0}^{v}=[1+\right.$ $\left.\left.\left(\rho^{v}\right)^{2}\right] N_{0}\right\}_{v=1}^{2 M_{A}-1}$, whereas $\left\{L_{a}(b \underset{m}{\sim})\right\}_{\underset{m}{m}=1}^{\bar{m}}$ denotes the a priori LLRs obtained from channel decoding. Similarly, the lowcomplexity Max-Log-MAP algorithm is formulated as [12]

$$
L_{p}\left(b_{m}\right)=\max _{s_{k} \in \mathbf{s}_{b_{m}=1}}\left[d\left(\rho^{v}, \omega^{l}\right)\right]-\max _{s_{k} \in \mathbf{s}_{b_{m}}=0}\left[d\left(\rho^{v}, \omega^{l}\right)\right]
$$

where only the maximum a posteriori probability metrics are taken into account.

It can be seen that the Log-MAP of (13) and the Max-LogMAP of (15) invoked by the JAPD have to evaluate $\left(2 M_{A}-\right.$ 1) $M_{P}$ metrics $\left\{\left\{d\left(\rho^{v}, \omega^{l}\right)\right\}_{v=1}^{2 M_{A}-1}\right\}_{l=1}^{M_{P}}$ of (14) to produce a single soft-bit decision. By contrast, the SAPD defines its amplitude-related probability metric $\left\{d\left(\rho^{v}\right)\right\}_{v=1}^{2 M_{A}-1}$ and phaserelated probability metric $\left\{d\left(\omega^{l}\right)\right\}_{l=1}^{M_{P}}$ separately as [13]

$$
\begin{aligned}
d\left(\rho^{v}\right)= & \sum_{n=1}^{N_{R}}\left[\frac{-\left|y_{k+1}^{n}\right|\left|y_{k}^{n}\right|}{N_{0}\left(1+\left(\lambda_{k}^{n}\right)^{2}\right)}\left(\lambda_{k}^{n}-\rho^{v}\right)^{2}\right] \\
& +\sum_{\widetilde{m}=\bar{m}_{P}+1}^{m} b_{\widetilde{m}} L_{a}(b \tilde{m})
\end{aligned}
$$

\footnotetext{
${ }^{1}$ The SAPD refers to the final results of partially combined differential detection (PDD) in [13]. Although the PDD's phase detector makes use of the received symbols' amplitudes, PDD still evaluates the $\left(2 M_{A}-1\right)$-sized amplitude subset and the $M_{P}$-sized phase subset separately for detecting the $\bar{m}_{A}$ and $\bar{m}_{P}$ bits, respectively. Therefore, PDD in [13] is referred to as SAPD in this paper.
}

$$
\begin{aligned}
d\left(\omega^{l}\right)=\sum_{n=1}^{N_{R}}[- & \frac{\left|y_{k+1}^{n}\right|^{2}+\left|y_{k}^{n}\right|^{2}\left(\lambda_{k}^{n}\right)^{2}}{N_{0}\left(1+\left(\lambda_{k}^{n}\right)^{2}\right)} \\
& \left.+\frac{2\left|y_{k+1}^{n}\right|\left|y_{k}^{n}\right| \lambda_{k}^{n} \cos \left(\Delta \theta_{k}^{n}-\angle \omega^{l}\right)}{N_{0}\left(1+\left(\lambda_{k}^{n}\right)^{2}\right)}\right] \\
& +\sum_{\widetilde{m}=1}^{\bar{m}_{P}} b_{\widetilde{m}} L_{a}\left(b_{\widetilde{m}}\right)
\end{aligned}
$$

where $\left\{y_{k}^{n}\right\}_{n=1}^{N_{R}}$ denotes the $n$th element in $\mathbf{y}_{k}$, whereas we have $\lambda_{k}^{n}=\left|y_{k+1}^{n}\right| /\left|y_{k}^{n}\right|$ and $\Delta \theta_{k}^{n}=\angle y_{k+1}^{n}-\angle y_{k}^{n}$. The LogMAP of (13) and the Max-Log-MAP of (15) invoked by the SAPD have only to evaluate and compare $\left(2 M_{A}-1\right)$ metrics $\left\{d\left(\rho^{v}\right)\right\}_{v=1}^{2 M_{A}-1}$ of (16a) and $M_{P}$ metrics $\left\{d\left(\omega^{l}\right)\right\}_{l=1}^{M_{P}}$ of $(16 \mathrm{~b})$ for producing the amplitude bit decisions and the phase bit decisions. As a result, the SAPD exhibits lower complexity.

If we use the average mutual information (AMI) as a measure of detection capability, then the AMI achieved by the JAPD may be expressed as [13], [22]

$$
\begin{aligned}
C_{\mathrm{JAPD}}= & I\left(\mathbf{y}_{k+1} \mid \mathbf{y}_{k} ; s_{k}\right) \\
= & \frac{1}{M M_{A}} \sum_{w=1}^{M_{A}} \sum_{u=1}^{M} E\left\{\log _{2}\left[\frac{M \cdot p\left(\mathbf{y}_{k+1} \mid \mathbf{y}_{k}, \bar{s}^{u}\right)}{\sum_{\bar{u}=1}^{M} p\left(\mathbf{y}_{k+1} \mid \mathbf{y}_{k}, \bar{s}^{\bar{u}}\right)}\right]\right. \\
& \left.\mid s_{k}=\bar{s}^{u}, \gamma_{k}=\gamma^{w}\right\}
\end{aligned}
$$

where $\left\{\gamma^{w}\right\}_{w=1}^{M_{A}}$ is taken from the $M_{A}$-element ring amplitude set $\gamma$ for $\gamma_{k}$, whereas $\left\{\bar{s}^{u}\right\}_{u=1}^{M}$ is taken from the $M$-element DAPSK symbol set $\overline{\mathbf{s}}$, whose size is smaller than the full set $\mathbf{s}$, simply because we have already decided upon the previous transmitted DAPSK symbol's amplitude $\left(\gamma_{k}=\gamma^{w}\right)$. Based on the received signal model of (5), the conditional probability seen in (17) is given by

$$
p\left(\mathbf{y}_{k+1} \mid \mathbf{y}_{k}, \bar{s}^{u}\right)=\frac{\exp \left[-\frac{\left\|\mathbf{y}_{k+1}-\bar{s}^{u} \mathbf{y}_{k}\right\|^{2}}{\left(1+\left|\bar{s}^{u}\right|^{2}\right) N_{0}}\right]}{\left[\pi\left(1+\left|\bar{s}^{u}\right|^{2}\right) N_{0}\right]^{N_{R}}} .
$$

Similarly, the AMI achieved by the SAPD [13], [22] is given by (19), shown at the bottom of the next page, where conditions $\left(\gamma_{k}=\gamma^{w_{1}}, \gamma_{k+1}=\gamma^{w_{2}}\right)$ and $\left(\gamma_{k}=\gamma^{w_{1}}, \gamma_{k+1}=\gamma^{\bar{w}_{2}}\right)$ determine amplitude variables $\rho^{v}$ and $\rho^{\bar{v}}$, respectively, whereas the conditional probabilities in (19) are given by (20), also shown at the bottom of the next page.

Based on (17) and (19), the AMI achieved by the JAPD and the SAPD is shown in Fig. 3. It can be seen that the JAPD and the SAPD have similar detection capabilities when we have $\left(N_{R}=1\right)$. However, the JAPD is capable of achieving a higher AMI, when multiple receive antennas are employed, as evidenced in Fig. 3 both for the 16-DAPSK $(2,8)$ scheme and for the 64-DAPSK $(4,16)$ arrangement.

\section{B. Reduced-Complexity Soft-Decision-Aided DAPSK Detection}

Since the a posteriori probability metrics $d\left(\rho^{v}, \omega^{l}\right)$ of (14) directly relate the symbol-level channel output 


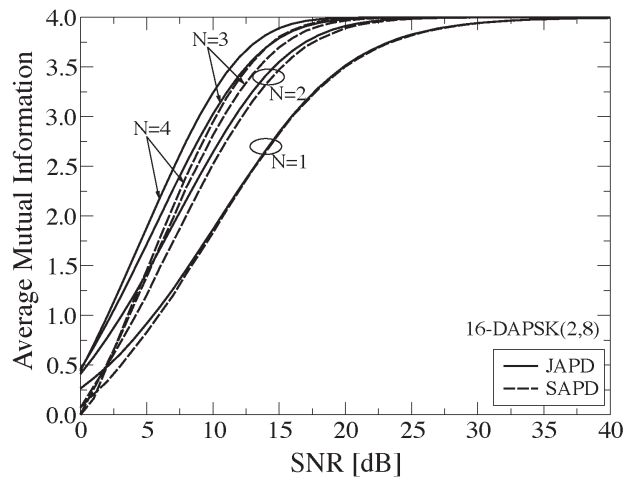

(a)

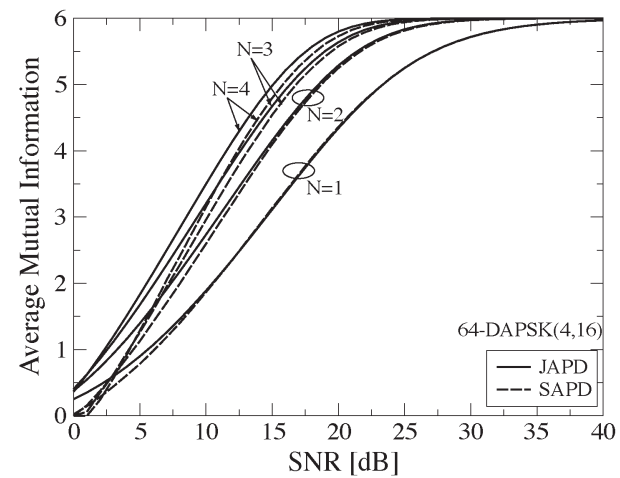

(b)

Fig. 3. Comparison between AMI achieved by the JAPD in [14] and AMI achieved by the SAPD in [13]. The system parameters are summarized in Table I. (a) 16-DAPSK $(2,8)$. (b) 64-DAPSK $(4,16)$.

$\left(-\left\|\mathbf{y}_{k+1}-\rho^{v} \omega^{l} \mathbf{y}_{k}\right\|^{2} / \tilde{N}_{0}^{v}\right)$ to the symbol-level a priori LLRs $\left[\sum_{\tilde{m}=1}^{\bar{m}} b \underset{m}{\sim} L_{a}(b \underset{m}{\sim})\right]$, the JAPD in Section III-A operates on a symbol-by-symbol basis, which implies that all of the DAPSK constellation points have to be visited by the JAPD. Observe in Section II-B that instead of evaluating all the DAPSK constellation points using (6), the hard-decision-aided DAPSK detection of (9) and (12) tests only a reduced subset of the constellation points. Furthermore, the Max-Log-MAP of (15) aims at finding the maximum metric, which is similar to the action of the harddecision-aided detection of (6). Motivated by this, here, we aim for linking each a priori soft-input bit to a specific part of the channel's output, so that only a reduced subset of the constellation points has to be evaluated by the JAPD. In the rest of this paper, we refer to the proposed Max-Log-MAP-aided DAPSK detection as the reduced-complexity JAPD (RC-JAPD).
Considering 16-DAPSK $(2,8)$ in Fig. 1 as our example, the Max-Log-MAP of (15) invoked for detecting the last bit $b_{4}$, which determines the ring amplitude, may be expressed as

$$
\begin{aligned}
L_{p}\left(b_{4}\right) & =\max _{b_{4}=1} d\left(\rho^{v}, \omega^{l}\right)-\max _{b_{4}=0} d\left(\rho^{v}, \omega^{l}\right) \\
& =\max _{\omega^{l} \in \boldsymbol{\omega}}\left\{d\left(\alpha^{-1}, \omega^{l}\right), d\left(\alpha, \omega^{l}\right)\right\}-\max _{\omega^{l} \in \boldsymbol{\omega}} d\left(1, \omega^{l}\right) \\
& =\max \left\{d_{\max }\left(\rho^{1}\right), d_{\max }\left(\rho^{3}\right)\right\}-d_{\max }\left(\rho^{2}\right)
\end{aligned}
$$

where the local maximum probability metric of a specific ring amplitude index $v \in\left\{1, \ldots, 2 M_{A}-1\right\}$ is given by

$$
d_{\max }\left(\rho^{v}\right)=\max _{\omega^{l} \in \boldsymbol{\omega}} d\left(\rho^{v}, \omega^{l}\right) .
$$

$$
\begin{aligned}
C_{\mathrm{SAPD}}= & I\left(\left\{\lambda_{k}^{n}\right\}_{n=1}^{N_{R}} ; \rho^{v}\right)+I\left(\left\{\Delta \theta_{k}^{n}, \lambda_{k}^{n}\right\}_{n=1}^{N_{R}} ; \omega^{l}\right) \\
= & \frac{1}{\left(M_{A}\right)^{2}} \sum_{w_{1}=1}^{M_{A}} \sum_{w_{2}=1}^{M_{A}} E\left\{\log _{2}\left[\frac{M_{A} \cdot p\left(\left\{\lambda_{k}^{n}\right\}_{n=1}^{N_{R}} \mid \rho^{v}\right)}{\sum_{\bar{w}_{2}=1}^{M_{A}} p\left(\left\{\lambda_{k}^{n}\right\}_{n=1}^{N_{R}} \mid \rho^{\bar{v}}\right)}\right] \mid \gamma_{k+1}=\gamma^{w_{2}}, \gamma_{k}=\gamma^{w_{1}}\right\} \\
& +\frac{1}{M} \sum_{w=1}^{M_{A}} \sum_{l=1}^{M_{P}} E\left\{\log _{2}\left[\frac{M_{P} \cdot p\left(\left\{\Delta \theta_{k}^{n}, \lambda_{k}^{n}\right\}_{n=1}^{N_{R}} \mid \omega^{l}\right)}{\sum_{\bar{l}=1}^{M_{P}} p\left(\left\{\Delta \theta_{k}^{n}, \lambda_{k}^{n}\right\}_{n=1}^{N_{R}} \mid \omega^{\bar{l}}\right)}\right] \mid \omega_{k}=\omega^{l}, \gamma_{k}=\gamma^{w}\right\}
\end{aligned}
$$

$$
\begin{aligned}
p\left(\left\{\lambda_{k}^{n}\right\}_{n=1}^{N_{R}} \mid \rho^{v}\right)= & \prod_{n=1}^{N_{R}}\left\{\frac{N_{0} \lambda_{k}^{n}\left[1+\left(\lambda_{k}^{n}\right)^{2}\right]+\lambda_{k}^{n}\left|y_{k}^{n}\right|^{2}\left(\rho^{v} \lambda_{k}^{n}+1\right)^{2}}{\left.\left[1+\left(\lambda_{k}^{n}\right)^{2}\right]^{\frac{5}{2}} \sqrt{\pi N_{0}\left|y_{k}^{n}\right|^{2} \lambda_{k}^{n} \rho^{v}} \exp \left[\frac{-\left|y_{k}^{n}\right|^{2}}{N_{0}\left(1+\left(\lambda_{k}^{n}\right)^{2}\right)}\left(\lambda_{k}^{n}-\rho^{v}\right)^{2}\right]\right\}}\right. \\
p\left(\left\{\Delta \theta_{k}^{n}, \lambda_{k}^{n}\right\}_{n=1}^{N_{R}} \mid \omega^{l}\right)= & \prod_{n}^{N_{R}}\left\{\frac{\lambda_{k}^{n}}{\pi\left[1+\left(\lambda_{k}^{n}\right)^{2}\right]^{2}}\left[1+\frac{\left|y_{k+1}^{n}\right|^{2}\left(\lambda_{k}^{n}\right)^{2}+\left|y_{k}^{n}\right|^{2}+2 \lambda_{k}^{n}\left|y_{k+1}^{n}\right|\left|y_{k}^{n}\right| \cos \left(\Delta \theta_{k}^{n}-\angle \omega^{l}\right)}{N_{0}\left(1+\left(\lambda_{k}^{n}\right)^{2}\right)}\right]\right. \\
& \left.\times \exp \left[-\frac{\left|y_{k+1}^{n}\right|^{2}+\left|y_{k}^{n}\right|^{2}\left(\lambda_{k}^{n}\right)^{2}-2\left|y_{k+1}^{n}\right|\left|y_{k}^{n}\right| \lambda_{k}^{n} \cos \left(\Delta \theta_{k}^{n}-\angle \omega^{l}\right)}{N_{0}\left(1+\left(\lambda_{k}^{n}\right)^{2}\right)}\right]\right\}
\end{aligned}
$$


Similar to the hard-decision metric simplifications seen in (7), the a posteriori probability metric of (14) may be further extended as

$$
\begin{aligned}
d\left(\rho^{v}, \omega^{l}\right)= & -\frac{\left\|\mathbf{y}_{k+1}\right\|^{2}}{\widetilde{N}_{0}^{v}}-\frac{\left(\rho^{v}\right)^{2}\left\|\mathbf{y}_{k}\right\|^{2}}{\widetilde{N}_{0}^{v}} \\
& +\frac{2 \rho^{v} \operatorname{Re}\left\{\left(\omega^{l}\right)^{*} \mathbf{y}_{k+1} \cdot \mathbf{y}_{k}^{*}\right)}{\widetilde{N}_{0}^{v}}+\sum_{\widetilde{m}=1}^{\bar{m}} b_{\widetilde{m}} L_{a}\left(b_{\widetilde{m}}\right) \\
= & -\frac{\left\|\mathbf{y}_{k+1}\right\|^{2}}{\widetilde{N}_{0}^{v}}-\frac{\left(\rho^{v}\right)^{2}\left\|\mathbf{y}_{k}\right\|^{2}}{\widetilde{N}_{0}^{v}}+\frac{2 \rho^{v} \operatorname{Re}\left(\omega^{l}\right)}{\widetilde{N}_{0}^{v}} \operatorname{Re}\left(\widetilde{z}_{k}\right) \\
& +\frac{2 \rho^{v} \operatorname{Im}\left(\omega^{l}\right)}{\widetilde{N}_{0}^{v}} \operatorname{Im}\left(\widetilde{z}_{k}\right)+\sum_{\widetilde{m}=1}^{\bar{m}} b_{\widetilde{m}} L_{a}\left(b_{\widetilde{m}}\right)
\end{aligned}
$$

where we have the new decorrelating variable of $\left(\widetilde{z}_{k}=\mathbf{y}_{k+1} \cdot \mathbf{y}_{k}^{*}\right)$. Therefore, the local maximum probability metric of (22) may be expressed as

$$
d_{\max }\left(\rho^{v}\right)=\left[\max _{\omega^{l} \in \boldsymbol{\omega}} \widetilde{d}\left(\rho^{v}, \omega^{l}\right)\right]+C_{v}
$$

where we explicitly relate amplitude index $v$ to the corresponding a priori $L_{a}\left(b_{4}\right)$ by defining

$$
C_{v}=-\frac{\left\|\mathbf{y}_{k+1}\right\|^{2}}{\widetilde{N}_{0}^{v}}-\frac{\left(\rho^{v}\right)^{2}\left\|\mathbf{y}_{k}\right\|^{2}}{\widetilde{N}_{0}^{v}}+b_{4} L_{a}\left(b_{4}\right)
$$

while the remaining phasor-related submetric is given by

$$
\begin{array}{r}
\widetilde{d}\left(\rho^{v}, \omega^{l}\right)=\frac{2 \rho^{v} \operatorname{Re}\left(\omega^{l}\right)}{\widetilde{N}_{0}^{v}} \operatorname{Re}\left(\widetilde{z}_{k}\right)+\frac{2 \rho^{v} \operatorname{Im}\left(\omega^{l}\right)}{\widetilde{N}_{0}^{v}} \operatorname{Im}\left(\widetilde{z}_{k}\right) \\
+\sum_{\widetilde{m}=1}^{\bar{m}_{P}} b_{\widetilde{m}} L_{a}\left(b_{\widetilde{m}}\right) .
\end{array}
$$

Let us now try to relate $L_{a}\left(b_{2}\right)$ and $L_{a}\left(b_{1}\right)$ to the real part and the imaginary part of decorrelating variable $\widetilde{z}_{k}$, respectively. For a specific ring amplitude index $v$, there are $\left(M_{P}=8\right)$ candidates for $\widetilde{d}\left(\rho^{v}, \omega^{l}\right)$. Considering the four candidates of $\omega^{l} \in\{\exp ( \pm j(\pi / 8)), \exp ( \pm j(7 \pi / 8))\}$, which share the same coordinate magnitudes but are associated with different signs, the resultant four candidates of $\widetilde{d}\left(\rho^{v}, \omega^{l}\right)$ seen in (26) may be expressed as

$$
\begin{aligned}
& \widetilde{d}\left(\rho^{v} \exp \left(j \frac{\pi}{8}\right)\right) \\
& \quad=\frac{2 \rho^{v} \cos \left(\frac{\pi}{8}\right)}{\widetilde{N}_{0}^{v}} \operatorname{Re}\left(\widetilde{z}_{k}\right)+\frac{2 \rho^{v} \sin \left(\frac{\pi}{8}\right)}{\widetilde{N}_{0}^{v}} \operatorname{Im}\left(\widetilde{z}_{k}\right) \\
& \quad=t_{\mathrm{Re}_{1}}^{v}+t_{\mathrm{Im}_{1}}^{v}+\frac{L_{a}\left(b_{1}\right)+L_{a}\left(b_{2}\right)}{2} \\
& \widetilde{d}\left(\rho^{v} \exp \left(j \frac{7 \pi}{8}\right)\right) \\
& \quad=-\frac{2 \rho^{v} \cos \left(\frac{\pi}{8}\right)}{\widetilde{N}_{0}^{v}} \operatorname{Re}\left(\widetilde{z}_{k}\right)+\frac{2 \rho^{v} \sin \left(\frac{\pi}{8}\right)}{\widetilde{N}_{0}^{v}} \operatorname{Im}\left(\widetilde{z}_{k}\right)+L_{a}\left(b_{2}\right)
\end{aligned}
$$

$$
\begin{aligned}
& =-t_{\mathrm{Re}_{1}}^{v}+t_{\operatorname{Im}_{1}}^{v}+\frac{L_{a}\left(b_{1}\right)+L_{a}\left(b_{2}\right)}{2} \\
& \widetilde{d}\left(\rho^{v} \exp \left(-j \frac{\pi}{8}\right)\right) \\
& =\frac{2 \rho^{v} \cos \left(\frac{\pi}{8}\right)}{\widetilde{N}_{0}^{v}} \operatorname{Re}\left(\widetilde{z}_{k}\right)-\frac{2 \rho^{v} \sin \left(\frac{\pi}{8}\right)}{\widetilde{N}_{0}^{v}} \operatorname{Im}\left(\widetilde{z}_{k}\right)+L_{a}\left(b_{1}\right) \\
& \quad=t_{\mathrm{Re}_{1}}^{v}-t_{\operatorname{Im}_{1}}^{v}+\frac{L_{a}\left(b_{1}\right)+L_{a}\left(b_{2}\right)}{2} \\
& \widetilde{d}\left(\rho^{v} \exp \left(-j \frac{7 \pi}{8}\right)\right) \\
& \quad=-\frac{2 \rho^{v} \cos \left(\frac{\pi}{8}\right)}{\widetilde{N}_{0}^{v}} \operatorname{Re}\left(\widetilde{z}_{k}\right) \\
& \quad-\frac{2 \rho^{v} \sin \left(\frac{\pi}{8}\right)}{\widetilde{N}_{0}^{v}} \operatorname{Im}\left(\widetilde{z}_{k}\right)+L_{a}\left(b_{1}\right)+L_{a}\left(b_{2}\right) \\
& =-t_{\operatorname{Re}_{1}}^{v}-t_{\operatorname{Im}_{1}}^{v}+\frac{L_{a}\left(b_{1}\right)+L_{a}\left(b_{2}\right)}{2}
\end{aligned}
$$

where we relate $\operatorname{Re}\left(\widetilde{z}_{k}\right)$ and $\operatorname{Im}\left(\widetilde{z}_{k}\right)$ to the corresponding a priori LLRs $L_{a}\left(b_{2}\right)$ and $L_{a}\left(b_{1}\right)$ by defining the following two test variables as

$$
\begin{aligned}
& t_{\mathrm{Re}_{1}}^{v}=\frac{2 \rho^{v} \cos \left(\frac{\pi}{8}\right)}{\widetilde{N}_{0}^{v}} \operatorname{Re}\left(\widetilde{z}_{k}\right)-\frac{L_{a}\left(b_{2}\right)}{2} \\
& t_{\operatorname{Im}_{1}}^{v}=\frac{2 \rho^{v} \sin \left(\frac{\pi}{8}\right)}{\widetilde{N}_{0}^{v}} \operatorname{Im}\left(\widetilde{z}_{k}\right)-\frac{L_{a}\left(b_{1}\right)}{2} .
\end{aligned}
$$

It may be seen in (27) that all four probability submetrics are constituted by three parts, i.e., they are $\left( \pm t_{\mathrm{Re}_{1}}^{v}\right),\left( \pm t_{\mathrm{Im}_{1}}^{v}\right)$, and a constant of $\left[L_{a}\left(b_{1}\right)+L_{a}\left(b_{2}\right) / 2\right]$. According to our arrangement, the only difference between the four candidates is the signs of the real and the imaginary test variables. Therefore, the local maximum submetric over $\omega^{l} \in\{\exp ( \pm j(\pi / 8))$, $\exp ( \pm j(7 \pi / 8))\}$ is directly given by

$$
\begin{aligned}
\tilde{d}_{\max _{1}}\left(\rho^{v}\right) & =\max _{\omega^{l} \in\left\{\exp \left( \pm j \frac{\pi}{8}\right), \exp \left( \pm j \frac{7 \pi}{8}\right)\right\}} \widetilde{d}\left(\rho^{v}, \omega^{l}\right) \\
& =\left|t_{\mathrm{Re}_{1}}^{v}\right|+\left|t_{\mathrm{Im}_{1}}^{v}\right|+\frac{L_{a}\left(b_{1}\right)+L_{a}\left(b_{2}\right)}{2} .
\end{aligned}
$$

Therefore, instead of evaluating and comparing a group of four probability submetrics in (27), the direct calculation of (29) may provide a significant $75 \%$ reduction in complexity.

Similarly, the other local maximum submetric over $\omega^{l} \in$ $\{\exp ( \pm j(3 \pi / 8)), \exp ( \pm j(5 \pi / 8))\}$ may be also given by a one-step calculation as

$$
\widetilde{d}_{\max _{2}}\left(\rho^{v}\right)=\left|t_{\mathrm{Re}_{2}}^{v}\right|+\left|t_{\mathrm{Im}_{2}}^{v}\right|+L_{a}\left(b_{3}\right)+\frac{L_{a}\left(b_{1}\right)+L_{a}\left(b_{2}\right)}{2}
$$


where the two new test variables are defined by

$$
\begin{aligned}
& t_{\mathrm{Re}_{2}}^{v}=\frac{2 \rho^{v} \sin \left(\frac{\pi}{8}\right)}{\widetilde{N}_{0}^{v}} \operatorname{Re}\left(\widetilde{z}_{k}\right)-\frac{L_{a}\left(b_{2}\right)}{2} \\
& t_{\mathrm{Im}_{2}}^{v}=\frac{2 \rho^{v} \cos \left(\frac{\pi}{8}\right)}{\widetilde{N}_{0}^{v}} \operatorname{Im}\left(\widetilde{z}_{k}\right)-\frac{L_{a}\left(b_{1}\right)}{2} .
\end{aligned}
$$

After considering all the $\left(M_{P}=8\right)$ phasors, the local maximum probability metric associated with a specific ring radius index $v$ seen in (22) is now given by

$$
d_{\max }\left(\rho^{v}\right)=\left[\max _{i \in\left\{1, \ldots, M_{P} / 4\right\}} \widetilde{d}_{\max _{i}}\left(\rho^{v}\right)\right]+C_{v}
$$

where $i \in\left\{1, \ldots, M_{P} / 4\right\}$ denotes the phasor index for the $M$-DAPSK constellation points in the first quadrant, and then, the global maximum metric pursued by the Max-Log-MAP of (21) may be obtained by invoking (32). Moreover, we note that $\left(L_{a}\left(b_{1}\right)+L_{a}\left(b_{2}\right)\right) / 2$ may be omitted in (29) and (30) because it is a common constant for all probability metrics $\left\{d_{\max }\left(\rho^{v}\right)\right\}_{v=1}^{2 M_{A}-1}$, and hence, it is eliminated by the negative polarity seen in the Max-Log-MAP of (21).

Since we have related $L_{a}\left(b_{2}\right)$ and $L_{a}\left(b_{1}\right)$ to the real and the imaginary parts of the decorrelating variable, the local maximum submetric over every set of four probability submetrics, which share the same magnitudes but are associated with different polarities, is obtained in a single step in (29) and (30). This implies that only the $\left(M_{P} / 4\right)$ specific phasors in the first quadrant have to be considered by the Max-Log-MAP, which is shown in Fig. 1.

More explicitly, we summarize the RC-JAPD proposed for the general $M$-DAPSK $\left(M_{A}, M_{P}\right)$ scheme as in Algorithm 1.

\section{Algorithm 1: RC-JAPD Detecting an $M$-DAPSK $\left(M_{A}, M_{P}\right)$ Symbol}

1) Update constants $\left\{C_{v}\right\}_{v=1}^{2 M_{A}-1}$, which relate the last $\bar{m}_{A}$ a priori LLRs $\left\{L_{a}\left(b_{\tilde{m}}\right)\right\}_{\tilde{m}=\bar{m}_{P}+1}^{\bar{m}}$ to ring amplitude index $v$ as

$$
C_{v}=-\frac{\left\|\mathbf{y}_{k+1}\right\|^{2}}{\widetilde{N}_{0}^{v}}-\frac{\left(\rho^{v}\right)^{2}\left\|\mathbf{y}_{k}\right\|^{2}}{\widetilde{N}_{0}^{v}}+\sum_{\widetilde{m}=\bar{m}_{P}+1}^{\bar{m}} b_{\widetilde{m}} L_{a}\left(b_{\tilde{m}}\right)
$$

where $\left(-\left\|\mathbf{y}_{k+1}\right\|^{2} / \widetilde{N}_{0}^{v}\right)$ only has to be estimated once, and $\left(-\left\|\mathbf{y}_{k}\right\|^{2} / \widetilde{N}_{0}^{v}\right)$ is known from detecting the previous received signal block.

2) Evaluate the test variables, which relate $L_{a}\left(b_{2}\right)$ and $L_{a}\left(b_{1}\right)$ to the real and imaginary parts of the decorrelating variable $\left(\widetilde{z}_{k}=\mathbf{y}_{k+1} \cdot \mathbf{y}_{k}^{*}\right)$ as

$$
\begin{aligned}
& t_{\operatorname{Re}_{i}}^{v}=\frac{2 \rho^{v} a_{i}}{\widetilde{N}_{0}^{v}} \operatorname{Re}\left(\widetilde{z}_{k}\right)-\frac{L_{a}\left(b_{2}\right)}{2} \\
& t_{\operatorname{Im}_{i}}^{v}=\frac{2 \rho^{v} b_{i}}{\widetilde{N}_{0}^{v}} \operatorname{Im}\left(\widetilde{z}_{k}\right)-\frac{L_{a}\left(b_{1}\right)}{2}
\end{aligned}
$$

where $\left\{\left(a_{i}, b_{i}\right)\right\}_{i=1}^{M_{P} / 4}$ are coordinates of the $M_{P}$-PSK phasors in the first quadrant.
3) Evaluate the local maximum submetric for each group, which relates the rest of the a priori LLRs $\left\{L_{a}\left(b_{\tilde{m}}\right)\right\}_{\tilde{m}=3}^{\bar{m}_{P}}$ to the $M_{P}$-PSK phasor index $i \in\left\{1, \ldots, M_{P} / 4\right\}$ as

$$
\widetilde{d}_{\max _{i}}\left(\rho^{v}\right)=\left|t_{\mathrm{Re}_{i}}^{v}\right|+\left|t_{\mathrm{Im}_{i}}^{v}\right|+\sum_{\widetilde{m}=3}^{\bar{m}_{P}} b_{\widetilde{m}} L_{a}\left(b_{\widetilde{m}}\right)
$$

so that the local maximum metric associated with a specific ring index $v$ may be obtained by

$$
d_{\max }\left(\rho^{v}\right)=\left[\max _{i \in\left\{1, \ldots, M_{P} / 4\right\}} \widetilde{d}_{\max _{i}}\left(\rho^{v}\right)\right]+C_{v} .
$$

4) The soft-bit output for the last $\bar{m}_{A}$ bits is directly given by

$$
\begin{aligned}
L_{p}\left(b_{m}\right)=\max _{b_{m}=1} d_{\max }\left(\rho^{v}\right)-\max _{b_{m}=0} & d_{\max }\left(\rho^{v}\right), \\
m & \in\left\{\bar{m}_{P}+1, \ldots, \bar{m}\right\}
\end{aligned}
$$

where the tentative indexes set for $\left[v \in\left\{1, \ldots, 2 M_{A}-1\right\}\right]$ is divided into two subsets corresponding to fixing the specific bit $b_{m}$ to 1 and 0 , respectively. Considering 16-DAPSK $(2,8)$ as an example, the subsets for index $v$ are given by $(v \in\{1,3\})$ and $(v=2)$, when we fix $b_{4}$ to 1 and 0 , respectively, as seen in (21).

5) When detecting the first two bits, which determine the quadrant, (37) is replaced by

$$
\begin{aligned}
& L_{p}\left(b_{m}\right)=\max _{b_{m}=1} d_{\max }\left(\rho^{v}\right)-\max _{b_{m}=0} d_{\max }\left(\rho^{v}\right), \\
& \quad m \in\{1,2\} .
\end{aligned}
$$

Furthermore, when a specific bit is fixed to $\left\{b_{m}=b\right\}_{m=1}^{2}$, where we have $b \in\{1,0\}$, the constellation set for $\omega^{l}$ is halved, and hence, Step 3 should be updated for (38) accordingly. For example, when $\left(b_{1}=1\right)$ is fixed, only the constellation points on the lower half of the constellation plane in Fig. 1 have to be considered, and hence, $\left|t_{\mathrm{Im}_{i}}^{v}\right|$ has to be replaced by $-t_{\operatorname{Im}_{i}}^{v}$ in (35). When $\left(b_{1}=0\right)$ is fixed, $\left|t_{\mathrm{Im}_{i}}^{v}\right|$ has to be replaced by $t_{\mathrm{Im}_{i}}^{v}$. Similarly, when the second bit $b_{2}$ is fixed to 1 or $0,\left|t_{\mathrm{Re}_{i}}^{v}\right|$ in (35) should be replaced by $-t_{\mathrm{Re}_{i}}^{v}$ or $t_{\mathrm{Re}_{i}}^{v}$, respectively.

6) When detecting the middle $\left(\bar{m}_{P}-2\right)$ bits, which determine the $\left(M_{P} / 4\right) M_{P}$-PSK phasors in the first quadrant, (37) may be replaced by

$$
\begin{aligned}
L_{p}\left(b_{m}\right)=\max _{b_{m}=1} d_{\max }\left(\rho^{v}\right)-\max _{b_{m}=0} d_{\max }\left(\rho^{v}\right), & \\
& m \in\left\{3, \ldots, \bar{m}_{P}\right\} .
\end{aligned}
$$

For a specific subset of $\omega^{l}$ defined by fixing $\left\{b_{m}=\right.$ $b\}_{m=3}^{\bar{m}_{P}}$, phasor index $i$ in (36) is updated as

$$
d_{\max }\left(\rho^{v}\right)=\left[\max _{b_{m}=b} \widetilde{d}_{\max _{i}}\left(\rho^{v}\right)\right]+C_{v}
$$

where the phasor index set of $\left(i \in\left\{1, \ldots, M_{P} / 4\right\}\right)$ is halved, when a specific bit $\left(b_{m}=b\right)$ is fixed. Considering 16-DAPSK $(2,8)$ as an example, we have $\left[d_{\max }\left(\rho^{v}\right)=\right.$ $\left.\widetilde{d}_{\max _{2}}\left(\rho^{v}\right)+C_{v}\right]$ for $(40)$ if $\left(b_{3}=1\right)$ is fixed, where only $(i=2)$ is considered. By contrast, only $(i=1)$ should be considered when $\left(b_{3}=0\right)$ is fixed, which results in the simple relationship of $\left[d_{\max }\left(\rho^{v}\right)=\widetilde{d}_{\max _{1}}\left(\rho^{v}\right)+C_{v}\right]$. 


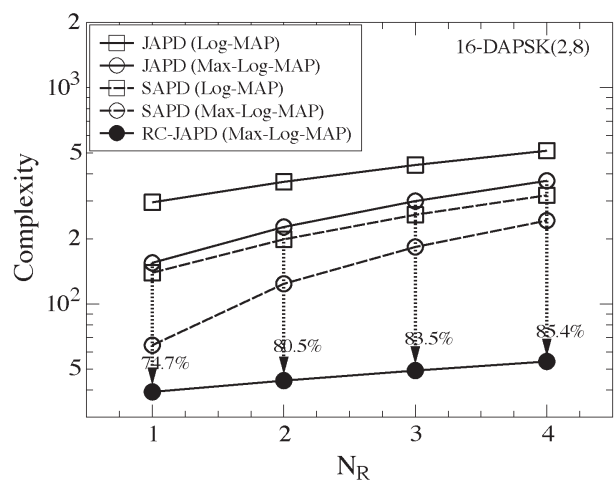

(a)

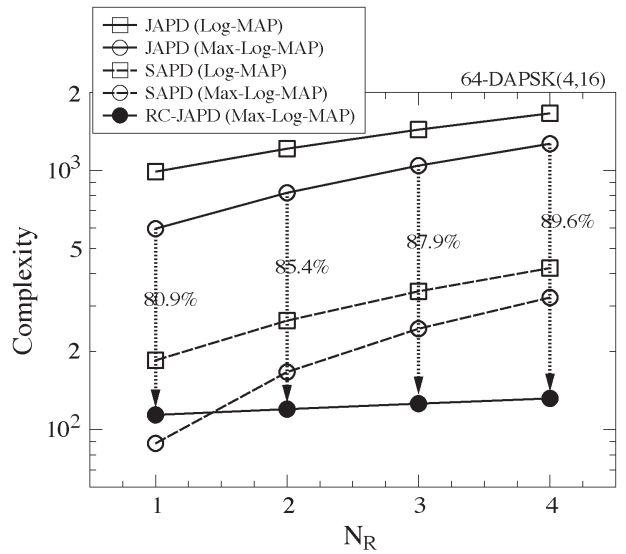

(b)

Fig. 4. Complexity comparison between the conventional JAPD (proposed in [14]) and the SAPD (proposed in [13]) in Section III-A and the proposed RC-JAPD in Section III-B. (a) 16-DAPSK(2,8). (b) 64-DAPSK $(4,16)$.

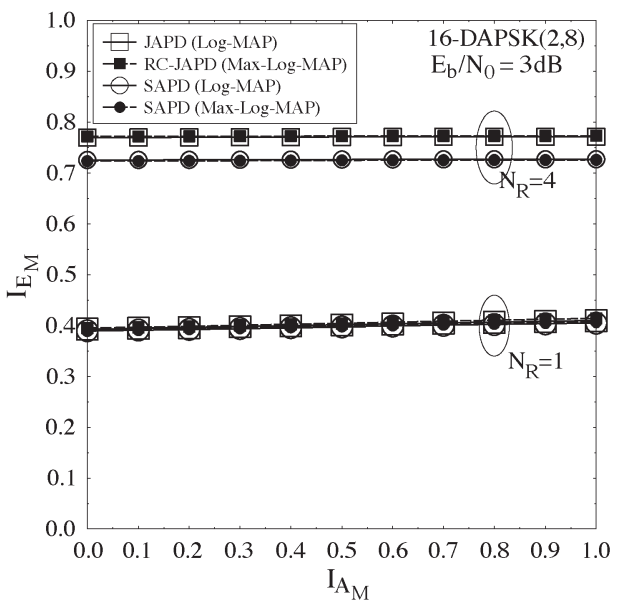

(a)

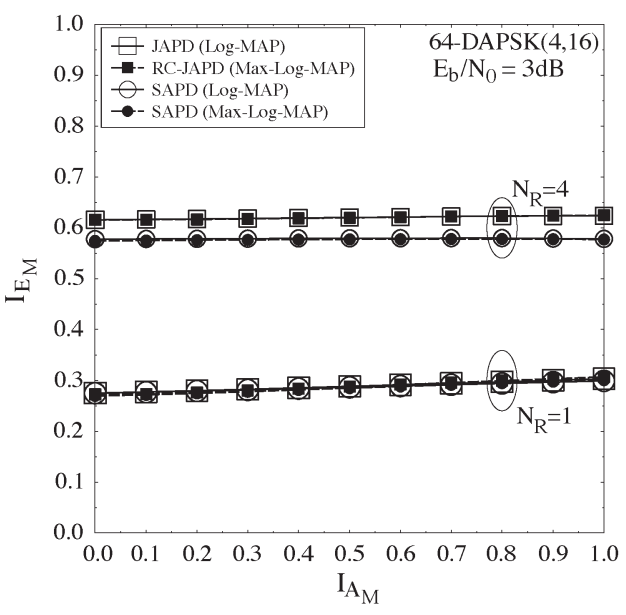

(b)

Fig. 5. EXIT charts of 16-DAPSK $(2,8)$ and 64-DAPSK $(4,16)$ invoking the JAPD, the SAPD, and the proposed RC-JAPD. (a) 16-DAPSK(2,8). (b) 64-DAPSK $(4,16)$.

\section{Complexity Analysis}

Here, we provide our complexity analysis for the three softdecision-aided DAPSK detectors in terms of both the number of constellation points visited by the detectors and the total number of real-valued calculations contributed by the detectors.

According to Section III-A, the total number of constellation points visited by the conventional JAPD is given by $\left[N_{v c}^{\mathrm{JAPD}}=\left(2 M_{A}-1\right) M_{P}\right]$. By contrast, the SAPD evaluates the amplitude subset and the phasor subset separately, so the total number of constellation points visited by the SAPD may be expressed by $\left[N_{v c}^{\mathrm{SAPD}}=\left(2 M_{A}-1\right)+M_{P}\right]$. Furthermore, as portrayed by Fig. 1, the proposed RC-JAPD visits a reduced number of the DAPSK constellation points, which is given by $\left[N_{v c}^{R C-J A P D}=\left(2 M_{A}-1\right) M_{P} / 4\right]$.

More specifically, for 16-DAPSK $(2,8)$, the JAPD visits $\left(N_{v c}^{\mathrm{JAPD}}=24\right)$ constellation points, which is higher than $\left(N_{v c}^{\mathrm{SAPD}}=11\right)$ of the SAPD, but the lowest is given by $\left(N_{v c}^{\mathrm{RC}-\mathrm{JAPD}}=6\right)$ of the proposed RC-JAPD. For $64-$ DAPSK $(4,16)$, our proposed RC-JAPD visits $\left(N_{v c}^{\mathrm{RC}-\mathrm{JAPD}}=\right.$ 28) constellation points, which is substantially lower than $\left(N_{v c}^{\mathrm{JAPD}}=112\right)$, but $N_{v c}^{\mathrm{RC}-\mathrm{JAPD}}$ is still slightly higher than $\left(N_{v c}^{\mathrm{SAPD}}=23\right)$.

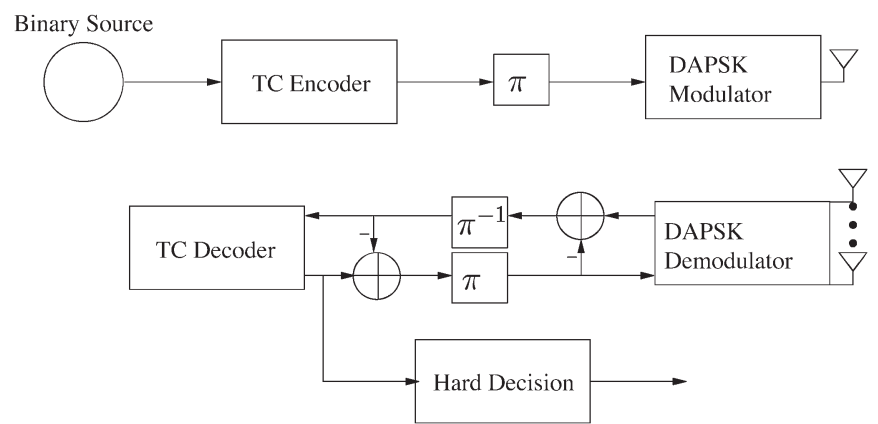

Fig. 6. Schematic of the TC-aided $M$-DAPSK $\left(M_{A}, M_{P}\right)$ scheme.

Since the DAPSK detectors are implemented by obeying different equations, we quantify the complexity in terms of the total number of real-valued calculations required for producing a single-bit decision. We note that the JAPD- and SAPD-aided DAPSK detection complexity increases multiplicatively as $N_{R}$ increases, owing to the fact that all the a posteriori probabilities at the $N_{R}$ antennas have to be multiplied together, as illustrated by (14) and (16). By contrast, the proposed RC-JAPD utilizes the decorrelating variable of $\left(\widetilde{z}_{k}=\mathbf{y}_{k+1} \cdot \mathbf{y}_{k}^{*}\right)$, which implies that all the detection procedures after Step 2 of Algorithm 1 
TABLE I

SYSTEM PARAMETERS

\begin{tabular}{|l|l|}
\hline Channel & Quasi-Static Rayleigh fading channels having a normalized Doppler frequency $\left(f_{d}\right)$ of 0.001. \\
\hline Frame length & 1000000 \\
\hline Quasi-Static symbol periods & $T_{Q S}=11$ \\
\hline Modulation & 16 -DAPSK $(2,8)$ and 64-DAPSK $(4,16)$. \\
\hline Mapping & Gray Mapping. \\
\hline \multirow{3}{*}{ Channel Coding } & (1) The parallel-concatenated Turbo Code (TC) of [25] \\
& (2) The serial-concatenated IRregular Convolutional Code (IRCC) of [23] amalgamated \\
& with Unity Rate Code (URC) of [26]. \\
\hline \multirow{2}{*}{ TC aided DAPSK scheme } & $I_{T C}=4$ iterations within TC, \\
& $I_{T C}-D A P S K=1$ iteration between TC decoder and 16-DAPSK(2,8) detector, \\
& $I_{T C-D A P S K}=2$ iterations between TC decoder and 64-DAPSK(4,16) detector. \\
\hline IRCC-URC aided & $I_{U R C-D A P S K}=1$ iteration between URC decoder and 16-DAPSK(2,8) detector, \\
DAPSK scheme & $I_{U R C-D A P S K}=2$ iterations between URC decoder and 64-DAPSK(4,16) detector, \\
& $I_{O u t}=50$ iterations between IRCC and the amalgamated URC-DAPSK decoder. \\
\hline
\end{tabular}

have exactly the same detection complexity as a single-antennabased detector.

We provide our complexity comparison between different DAPSK detectors in Fig. 4, which shows that the JAPD generally exhibits higher complexity than the SAPD. However, a significant $74.7 \%-89.6 \%$ complexity reduction is achieved by the proposed RC-JAPD compared with the JAPD using the Max-Log-MAP, both for 16-DAPSK $(2,8)$ and for 64$\operatorname{DAPSK}(4,16)$. As a result, for 16-DAPSK $(2,8)$ detection, the RC-JAPD imposes the lowest detection complexity, as evidenced in Fig. 4(a), owing to the fact that RC-JAPD visits the lowest number of constellation points in this case. Furthermore, observe in Fig. 4(b) for 64-DAPSK $(4,16)$ that the proposed RC-JAPD still exhibits slightly higher complexity than the SAPD using the Max-Log-MAP, when we have $\left(N_{R}=1\right)$, because $\left(N_{v c}^{\mathrm{RC}-\mathrm{JAPD}}=28\right)$ is higher than $\left(N_{v c}^{\mathrm{SAPD}}=23\right)$ for 64-DAPSK $(4,16)$. However, as $N_{R}$ increases, the complexity of the proposed RC-JAPD becomes the lowest again, as evidenced in Fig. 4(b). This is because the SAPD's complexity increases multiplicatively as $N_{R}$ increases, whereas only a part of RCJAPD's complexity is affected by $N_{R}$.

\section{Performance Results}

Our performance results are presented here. To investigate the extrinsic information transfer (EXIT) characteristics of the DAPSK detectors, we portray the EXIT charts [23] of both 16-DAPSK $(2,8)$ and 64-DAPSK $(4,16)$ in Fig. 5. It is shown that, similar to the hard-decision-aided DAPSK's performance in Fig. 2, the JAPD and the RC-JAPD exhibit an improved performance advantage over SAPD as $N_{R}$ increases, which is evidenced in Fig. 5. Furthermore, Fig. 5 shows that the performance difference between the Log-MAP and the MaxLog-MAP invoked by both the JAPD and the SAPD is marginal. Hence, employment of the Approx-Log-MAP [24], which corrects Max-Log-MAP's approximation with the aid of a correction term stored in a lookup table, is not neccessary for DAPSK detection. In this paper, we only apply the RC-JAPD and the SAPD relying on the Max-Log-MAP for the coded systems considered.

As shown in Fig. 6, the soft DAPSK detectors may be invoked by our iterative demapping-and-decoding-assisted turbo-coded (TC) [25] systems. The half-rate TC employed is constituted by two half-rate recursive convolutional codes associated with a constraint length of $K=3$ (using the octal gener-

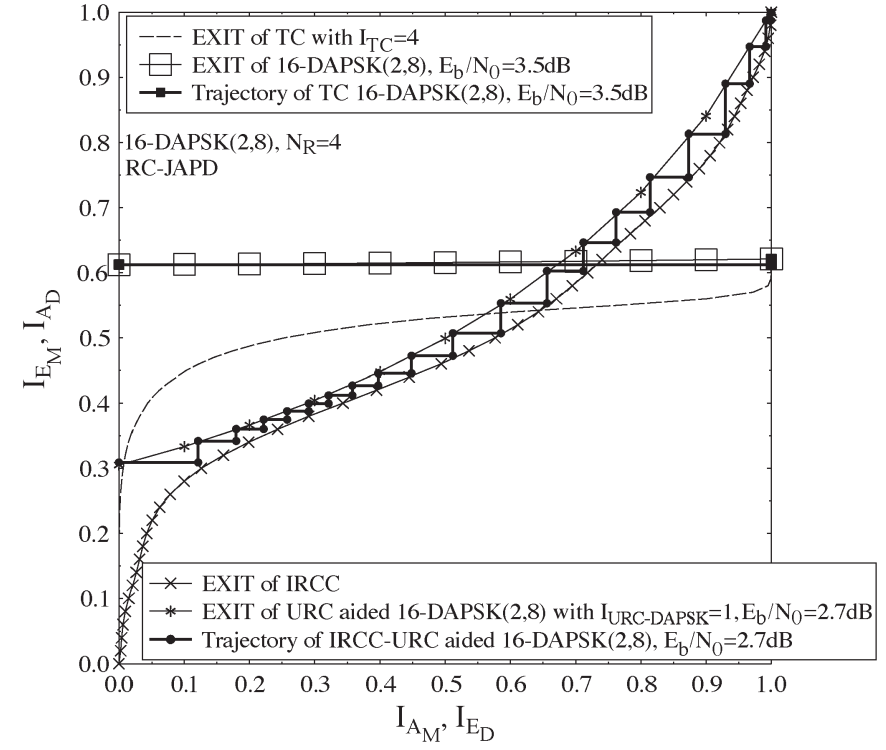

Fig. 7. EXIT charts and decoding trajectory for TC/IRCC-URC-aided 16-DAPSK $(2,8)$ detection, where the proposed RC-JAPD is invoked.

ator polynomials in $[5,7])$ and with the half-rate puncturing of the parity bits. To achieve further improved near-capacity performance, an irregular convolutional code (IRCC) in [23] amalgamated with the unity rate code (URC) in [26] and our DAPSK scheme may be conceived according to the schematic in [27]. We summarize our simulation parameters in Table I, where the number of iterations between the DAPSK detector and the TC/URC decoder was set to $\left(I_{\mathrm{TC}-\mathrm{DAPSK}} / I_{\mathrm{URC}-\mathrm{DAPSK}}=\right.$ $1)$ and $\left(I_{\text {TC-DAPSK }} / I_{\text {URC-DAPSK }}=2\right)$ for $16-\operatorname{DAPSK}(2,8)$ and 64-DAPSK $(4,16)$, respectively, because in contrast to 16 $\operatorname{DAPSK}(2,8)$, the 64-DAPSK $(4,16)$ scheme has a useful iteration gain, as shown in Fig. 5.

We portray the Monte-Carlo-simulation-based decoding trajectory in Fig. 7, which shows that the IRCC-URC-aided 16-DAPSK $(2,8)$ is capable of converging at a lower signal-tonoise ratio than the TC-aided 16-DAPSK $(2,8)$. The attainable bit-error-rate (BER) performance is shown in Fig. 8, where the AMI achieved by the JAPD and the SAPD are calculated according to (17) and (19), respectively. More explicitly, the AMI shown in Fig. 8 characterizes the DAPSK detector's capability in conjunction with half-rate channel coding, which may be quantified by the $E_{b} / N_{0}$ value, where the DAPSK detectors' AMI achieves half its maximum rate. It is shown in 


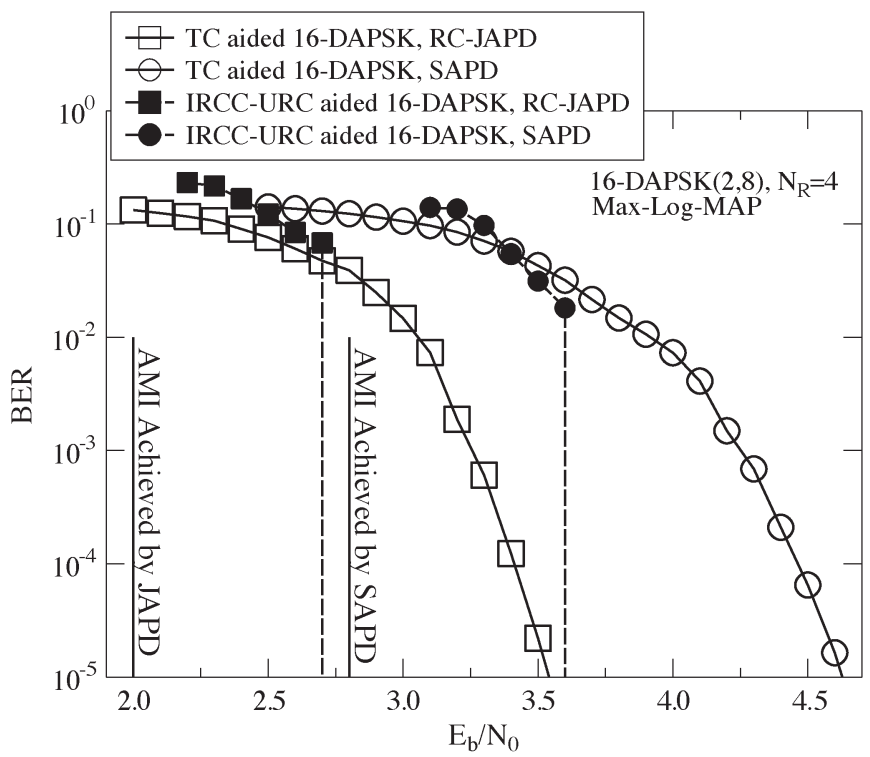

(a)

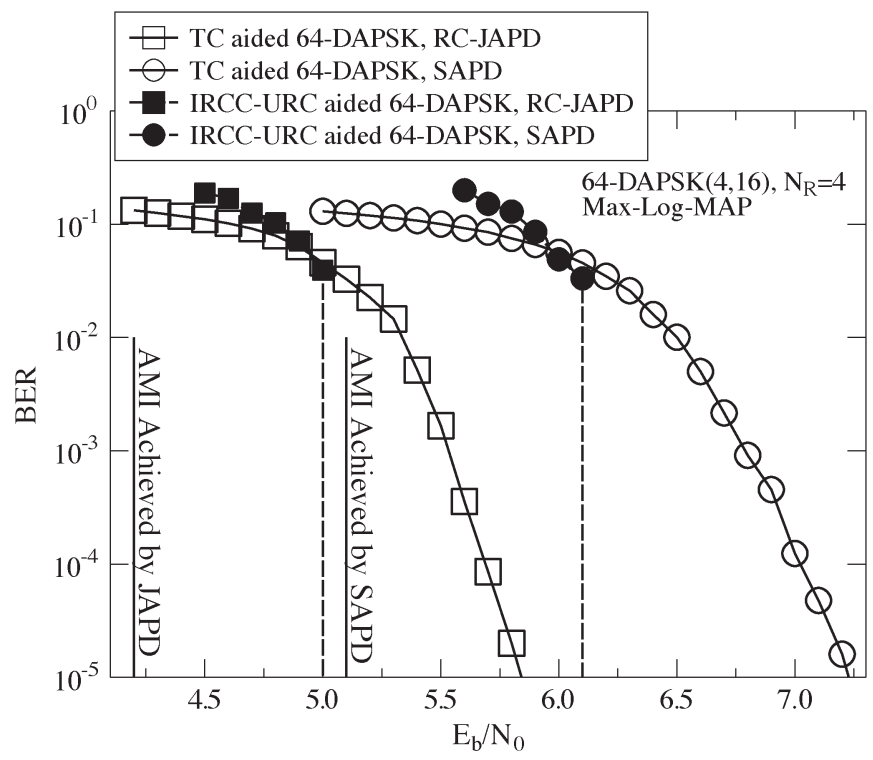

(b)

Fig. 8. BER performance of TC/IRCC-URC-aided 16-DAPSK $(2,8)$ and $64-D A P S K(4,16)$ detection, where the SAPD using Max-Log-MAP and the proposed RC-JAPD are invoked. (a) TC/IRCC-URC-aided 16-DAPSK(2,8). (b) TC/IRCC-URC-aided 64-DAPSK $(4,16)$.

Fig. 8 for both 16-DAPSK $(2,8)$ and 64-DAPSK $(4,16)$ that the proposed RC-JAPD outperforms the SAPD using the Max-LogMAP by $0.9-1.4 \mathrm{~dB}$, both for the TC-aided DAPSK scheme and for the IRCC-URC-aided DAPSK scheme, when $\left(N_{R}=4\right)$ receive antennas are employed.

\section{CONCLusion}

In this paper, we have demonstrated that separately detecting the ring amplitude and the phase of DAPSK imposes a performance loss, which is particularly significant for soft-decisionaided DAPSK detection invoked by iterative demapping and decoding schemes relying on multiple receive antennas. As a result, compared with the SAPD [13], the JAPD [14] has higher detection capability, but its detection complexity may become excessive. As a remedy, we proposed a new RC-JAPD, which links each a priori soft-input bit to a specific part of the channel's output, so that only a reduced subset of the DAPSK constellation points has to be evaluated by the soft DAPSK detector. Our simulation results demonstrate that the proposed RC-JAPD achieves further improved near-capacity performance (shown in Fig. 8), which is attained at reduced detection complexity (shown in Fig. 4), when multiple receive antennas are employed.

This contribution may be considered to be particularly beneficial for cooperative communications systems [8], [19], [20], which may employ DAPSK to dispense with channel estimation at the relay nodes.

\section{REFERENCES}

[1] S. ten Brink, J. Speidel, and R.-H. Yan, "Iterative demapping and decoding for multilevel modulation," in Proc. IEEE GLOBECOM, Nov. 1998, vol. 1, pp. 579-584.

[2] E. Issman and W. Webb, "Carrier recovery for 16 level QAM in mobile radio," in Proc. IEE Colloquium Multi-Level Modul. Tech. Point-to-Point Mobile Radio, Mar. 1990, pp. 9/1-9/8.

[3] L. Hanzo, S. X. Ng, T. Keller, and W. Webb, Quadrature Amplitude Modulation: From Basics to Adaptive Trellis-Coded, Turbo-Equalised and Space-Time Coded OFDM, CDMA and MC-CDMA Systems Digital Communications., 2nd ed. Hoboken, NJ, USA: Wiley-IEEE Press, 2004.

[4] L. Chen, H. Kusaka, and M. Kominami, "Blind phase recovery in QAM communication systems using higher order statistics," IEEE Signal Process. Lett., vol. 3, no. 5, pp. 147-149, May 1996.

[5] Y. Wang and E. Serpedin, "A class of blind phase recovery techniques for higher order QAM modulations: Estimators and bounds," IEEE Signal Process. Lett., vol. 9, no. 10, pp. 301-304, Oct. 2002.

[6] H. Rohling and V. Engels, "Differential amplitude phase shift keying (DAPSK) - A new modulation method for DTVB," in Proc. IBC, Sep. 1995, pp. 102-108.

[7] W. Webb, L. Hanzo, and R. Steele, "Bandwidth efficient QAM schemes for Rayleigh fading channels," Proc. Inst. Elect. Eng I-Commun. Speech Vis., vol. 138, no. 3, pp. 169-175, Jun. 1991.

[8] L. Wang and L. Hanzo, "Dispensing with channel estimation: Differentially modulated cooperative wireless communications," IEEE Commun. Surveys Tuts., vol. 14, no. 3, pp. 836-857, 3rd Quart., 2012.

[9] S. ten Brink, J. Speidel, and R.-H. Han, "Iterative demapping for QPSK modulation," Electron. Lett., vol. 34, no. 15, pp. 1459-1460, Jul. 1998.

[10] L. Bahl, J. Cocke, F. Jelinek, and J. Raviv, "Optimal decoding of linear codes for minimizing symbol error rate," IEEE Trans. Inf. Theory, vol. IT20, no. 2, pp. 284-287, Mar. 1974.

[11] J. Hagenauer and P. Hoeher, "A Viterbi algorithm with soft-decision outputs and its applications," in Proc. IEEE GLOBECOM, Nov. 1989, vol. 3, pp. 1680-1686.

[12] W. Koch and A. Baier, "Optimum and sub-optimum detection of coded data disturbed by time-varying intersymbol interference," in Proc. IEEE GLOBECOM, Dec. 1990, vol. 3, pp. 1679-1684.

[13] K. Ishibashi, H. Ochiai, and R. Kohno, "Low-complexity bit-interleaved coded DAPSK for Rayleigh-fading channels," IEEE J. Sel. Areas Commun., vol. 23, no. 9, pp. 1728-1738, Sep. 2005.

[14] D. Liang, S. X. Ng, and L. Hanzo, "Soft-decision Star-QAM aided BICMID," IEEE Signal Process. Lett., vol. 18, no. 3, pp. 169-172, Mar. 2011.

[15] X. Dong, N. Beaulieu, and P. Wittke, "Error probabilities of twodimensional $M$-ary signaling in fading," IEEE Trans. Commun., vol. 47, no. 3, pp. 352-355, Mar. 1999.

[16] L.-J. Lampe and R. Fischer, "Comparison and optimization of differentially encoded transmission on fading channels," in Proc. ISPLC, 1999, pp. 107-113.

[17] T. May, H. Rohling, and V. Engels, "Performance analysis of Viterbi decoding for 64-DAPSK and 64-QAM modulated OFDM signals," IEEE Trans. Commun., vol. 46, no. 2, pp. 182-190, Feb. 1998.

[18] D. Liang, S. X. Ng, and L. Hanzo, "Near-capacity turbo coded softdecision aided DAPSK/Star-QAM," in Proc. IEEE VTC Fall, Sep. 2011, pp. 1-5.

[19] J. Laneman and G. Wornell, "Distributed space-time-coded protocols for exploiting cooperative diversity in wireless networks," IEEE Trans. Inf. Theory, vol. 49, no. 10, pp. 2415-2425, Oct. 2003. 
[20] J. Laneman, D. Tse, and G. Wornell, "Cooperative diversity in wireless networks: Efficient protocols and outage behavior," IEEE Trans. Inf. Theory, vol. 50, no. 12, pp. 3062-3080, Dec. 2004.

[21] L. Hanzo, O. Alamri, N. El-Hajjar, and N. Wu, Near-Capacity Multi Functional MIMO Systems. Hoboken, NJ, USA: Wiley, May 2009.

[22] S. X. Ng and L. Hanzo, "On the MIMO channel capacity of multidimensional signal sets," IEEE Trans. Veh. Technol., vol. 55, no. 2, pp. 528-536, Mar. 2006.

[23] M. Tuchler, "Design of serially concatenated systems depending on the block length," IEEE Trans. Commun., vol. 52, no. 2, pp. 209-218, Feb. 2004.

[24] P. Robertson, E. Villebrun, and P. Hoeher, “A comparison of optimal and sub-optimal MAP decoding algorithms operating in the log domain," in Proc. IEEE ICC, Jun. 1995, vol. 2, pp. 1009-1013.

[25] C. Berrou and A. Glavieux, "Near optimum error correcting coding and decoding: Turbo-codes," IEEE Trans. Commun., vol. 44, no. 10, pp. 1261-1271, Oct. 1996.

[26] H. Tullberg and P. Siegel, "Serial concatenated TCM with an inner accumulate code-Part I: Maximum-likelihood analysis," IEEE Trans. Commun., vol. 53, no. 1, pp. 64-73, Jan. 2005.

[27] C. Xu, S. X. Ng, and L. Hanzo, "Near-capacity irregular convolutional coded cooperative differential linear dispersion codes using multiplesymbol differential detection," IEEE Signal Process. Lett., vol. 18, no. 3, pp. 173-176, Mar. 2011.

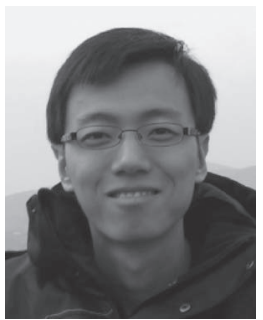

Chao Xu (S'09) received the B.Eng. degree from Beijing University of Posts and Telecommunications, Beijing, China, and the B.S.E. degree (first-class honors) from Queen Mary, University of London, London, U.K., in 2008, both in telecommunications engineering with management and both through a Sino-U.K. joint degree program, and the M.Sc. degree (with distinction) in radio frequency communication systems from the University of Southampton, Southampton, U.K., in 2009. He is currently working toward the Ph.D. degree with the Research Group of Communications, Signal Processing and Control, School of Electronics and Computer Science, University of Southampton.

His research interests include reduced-complexity multiple-input-multipleoutput design, noncoherent space-time modulation detection, extrinsicinformation-transfer-chart-aided turbo detection, and cooperative communications.

Mr. Xu was awarded the Best M.Sc. Student in Broadband and Mobile Communication Networks by the IEEE Communications Society (United Kingdom and Republic of Ireland Chapter).

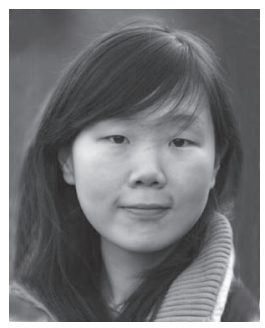

Dandan Liang (S'12) received the B.Eng. degree (first class) in electronic science and technology from the PLA Information Engineering University, Zhengzhou, China, in 2008 and the M.Sc. degree (first class) in radio frequency communication systems from the University of Southampton, Southampton, U.K., in 2009. She is currently working toward the Ph.D. degree with the Research Group of Communications, Signal Processing and Control, School of Electronics and Computer Science, University of Southampton.

Her research interests include adaptive coded modulation, coded modulation, noncoherent/coherent modulation detection, iterative detection, network coding, cooperative communications, and wireless-optical fiber communications.

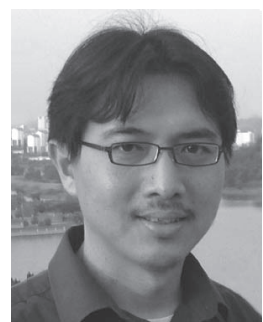

Soon Xin Ng (S'99-M'03-SM'08) received the B.Eng. degree (first class) in electronics engineering and the Ph.D. degree in wireless communications from the University of Southampton, Southampton, U.K., in 1999 and 2002, respectively.

From 2003 to 2006, he was a Postdoctoral Research Fellow working on collaborative European research projects including SCOUT, NEWCOM, and PHOENIX. Since August 2006, he has been a member of the academic staff with the School of Electronics and Computer Science, University of Southampton. He is involved in the OPTIMIX and CONCERTO European projects and the India-U.K. Advanced Technology Centre and U.K.-China Science Bridges $\mathrm{R} \& \mathrm{D}$ on (B) $4 \mathrm{G}$ Wireless Mobile Communications projects. He is currently a Senior Lecturer with the University of Southampton. He has published over 150 papers and coauthored two John Wiley/IEEE Press books. His research interests include adaptive coded modulation, coded modulation, channel coding, space-time coding, joint source-and-channel coding, iterative detection, orthogonal frequency-division multiplexing, multiple-input multiple-output, cooperative communications, distributed coding, quantum error-correction codes, and joint wireless-and-optical-fiber communications.

Dr. Ng is a Chartered Engineer and a Fellow of the Higher Education Academy in the U.K.

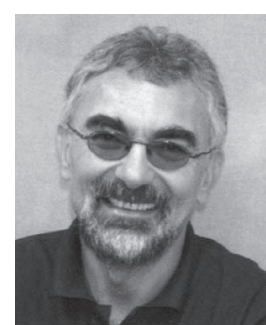

Lajos Hanzo (M'91-SM'92-F'04) received the M.S. degree (with first-class honors) in electronics and the Ph.D. degree from the Technical University of Budapest, Budapest. Hungary, in 1976 and 1983 , respectively, the D.Sc. degree from the University of Southampton, Southampton, U.K., in 2004, and the "Doctor Honoris Causa" degree from the Technical University of Budapest in 2009

During his 35-year career in telecommunications, he has held various research and academic posts in Hungary, Germany, and the U.K. Since 1986, he has been with the School of Electronics and Computer Science, University of Southampton, Southampton, U.K., where he holds the Chair for Telecommunications. Since 2009, he has been a Chaired Professor with Tsinghua University, Beijing China. He is currently directing a 100-strong academic research team, working on a range of research projects in the field of wireless multimedia communications sponsored by industry; the Engineering and Physical Sciences Research Council, U.K.; the European IST Programme; and the Mobile Virtual Centre of Excellence, U.K. He is an enthusiastic supporter of industrial and academic liaison and offers a range of industrial courses. He has successfully supervised $80 \mathrm{Ph}$.D. students, coauthored 20 John Wiley/IEEE Press books on mobile radio communications totaling in excess of 10000 pages, published more than 1250 research entries on IEEE Xplore, and presented keynote lectures. (For further information on research in progress and associated publications, please refer to http://www-mobile.ecs.soton.ac.uk.)

Dr. Hanzo is Fellow of the Royal Academy of Engineering, U.K., a Fellow of the Institution of Electrical Engineers, and a Governor of the IEEE Vehicular Technology Society. He has been a Technical Program Committee Chair and a General Chair for IEEE conferences. During 2008-2012, he was the Editor-inChief of the IEEE Press. He has received a number of distinctions. 